\title{
An Unusual Pattern of Peptide-bound Lysine Metabolism in Collagen from an Infant with Lethal Perinatal Osteogenesis Imperfecta
}

Olga M. Petrovic and Edward J. Miller Department of Medicine, Department of Biochemistry, and Institute of Dental Research, University of Alabama Medical Center, Birmingham, Alabama 35294

bstract. Collagens extracted from bones, cartilage, dermis, and dura mater of an infant with type II (lethal perinatal) osteogenesis imperfecta were evaluated with respect to chain composition and chemical characteristics of their constituent chains. The results indicated that the various types of collagen were present in the indicated tissues in proportions that approximated normal tissues. Nevertheless, the constituent chains of collagens extracted from dermis, i.e., $\alpha 1(\mathrm{I}), \alpha 2(\mathrm{I}), \alpha 1(\mathrm{III}), \alpha 1(\mathrm{~V})$, and $\alpha 2(\mathrm{~V})$, chromatographed on carboxymethyl cellulose as though they possessed substantially lower overall positive charge than the homologous chains of normal tissues. Amino acid analyses of the chains confirmed this observation and showed that the chains lacked five to seven residues of lysine (plus hydroxylysine). It was subsequently shown that the apparent deficiency in lysyl residues was due, at least in part, to the presence of unusually high levels of allysine, a cross-link precursor formed from peptide-bound lysine under the catalytic action of lysyl oxidase. These results, in conjunction with previous results obtained on collagens from type II osteogenesis imperfecta tissues, suggest that aberrant fibril formation in this syndrome allows increased lysyl oxidase activity.

\section{Introduction}

Osteogenesis imperfecta $(\mathrm{OI})^{1}$ is one of the most striking of the inherited disorders of bone and demonstrates marked genetic

Received for publication 15 August 1983 and in revised form 1 February 1984.

1. Abbreviations used in this paper: ASC, acid soluble collagen; CM-, carboxymethyl; CNBr, cyanogen bromide; HPLC, high performance liquid chromatography; NSSC, neutral salt soluble collagen; OI, osteogenesis imperfecta; PSC, pepsin-solubilized collagen.

J. Clin. Invest.

(c) The American Society for Clinical Investigation, Inc. 0021-9738/84/06/1569/07 \$1.00

Volume 73, June 1984, 1569-1575 and clinical heterogeneity. Patients vary greatly in the degree of bone fragility and may also manifest otosclerosis, dentinogenesis imperfecta, blue sclerae, cardiac valvular insufficiency, hernia, and easy skin bruisability. The spectrum of OI phenotypes has been divided into four major categories (1). Type II (lethal perinatal) $\mathrm{OI}$ is inherited as an autosomal recessive trait and occasionally arises by spontaneous mutation. It is the most severe form of OI, and affected infants have a markedly deficient bone mass and sustain multiple fractures beginning in utero that result in stillbirth or death early in the postnatal period.

A multitude of biochemical defects have been ascribed to the four different types of $\mathrm{OI}$ and have been reviewed in detail elsewhere (2-5). Reports on the biochemical defects associated with type II OI clearly reveal that this apparently single clinical entity may involve several different defects in collagen metabolism. Thus, an early report indicated that bone collagen of such an infant contained higher than normal levels of hydroxylysine (6). Subsequently it was shown that bone $\alpha 1$ (I) and $\alpha 2$ (I) chains, as well as cartilage $\alpha$ l(II) chains, contained markedly increased hydroxylysine levels, but that $\alpha 1$ (I) and $\alpha 2$ (I) chains from skin of the same infant showed only a small increase in hydroxylysine levels over that of controls (7). These results, with respect to the type I collagen chains of bone, have been confirmed in studies on an additional infant (8). Nevertheless, the latter study showed that $\alpha 1$ (I), $\alpha 2(\mathrm{I})$, and $\alpha 1$ (III) chains of dermis exhibited increased levels of hydroxylysine while the $\alpha 1$ (II) chains derived from cartilage collagen exhibited normal levels of hydroxylysine. A recent review contains brief reference to a study on a type II OI infant in which a decreased level of hydroxylysine was observed for bone collagen chains (2). Yet other studies on lethal perinatal OI bone have revealed a small but significant increase in the levels of type III and V collagens in the bone matrix (9). And finally, studies on skin fibroblasts derived from a type II OI infant have revealed a marked diminution in the ratio of type $\mathrm{I} /$ type III procollagen production (10). Additional studies on the same cell line showed that decreased type I procollagen secretion could be ascribed to intracellular retention of molecules that contain a structurally abnormal pro- $\alpha 1(\mathrm{I})$ chain $(11,12)$.

This paper reports yet another biochemical abnormality in the collagens of an infant exhibiting the characteristic features of lethal perinatal OI. The abnormality was detectable by the 
methods employed only in the collagen derived from the dermis, yet was common to all collagens recovered from the dermis, i.e., type I, III, and V.

\section{Methods}

The tissues studied in this investigation were from a female infant that was the product of an otherwise normal term pregnancy. Death occurred several hours after birth. Radiographs (Fig. 1) showed findings typical of type II OI including camptomelia, a markedly thin skull, beaded ribs, and multiple fractures. Sclerae were blue and the facies typical of OI, but no cutaneous or visceral abnormalities were apparent.

Specimens from dermis, dura mater, articular cartilage, skull bones, and long bones were carefully dissected, quickly rinsed in cold $\left(4^{\circ} \mathrm{C}\right)$ distilled water, and frozen until they were further processed for extraction and purification of collagens. The latter procedures were performed at $4^{\circ} \mathrm{C}$ essentially as described in a recent review (13). In brief, specimens of dermis and dura mater were extracted successively with the following solutions: $1.0 \mathrm{M} \mathrm{NaCl}, 0.05 \mathrm{M}$ Tris, $\mathrm{pH} 7.5 ; 0.5 \mathrm{M}$ acetic acid, $\mathrm{pH}$ 2.5; and $0.5 \mathrm{M}$ acetic acid, pH 2.5, containing sufficient pepsin (PM, Worthington Diagnostic Systems, Inc., Freehold, N.J.) to equal one-tenth the dry weight of tissue. The collagen present in each extract was purified and fractionated by selective salt precipitation from acidic (14) and neutral (15) solvents in order to isolate the various types of collagen

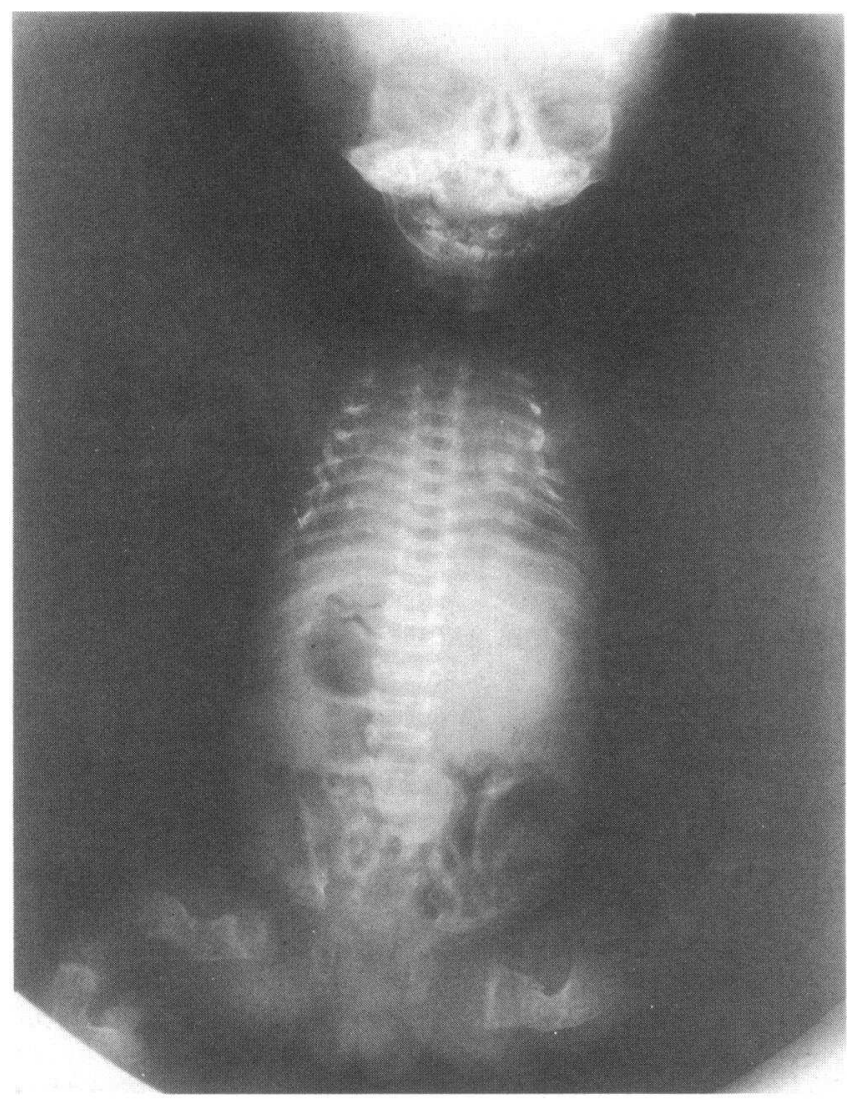

Figure 1. Radiograph of OI infant demonstrating deficient skull ossification, beaded ribs, camptomelia, and multiple fractures. present. Specimens of cartilage were extracted with $4.0 \mathrm{M}$ guanidine$\mathrm{HCl}, 0.05 \mathrm{M}$ Tris, $\mathrm{pH} 7.5$, to remove proteoglycans before limited pepsin digestion in $0.5 \mathrm{M}$ acetic acid, and collagen extracted from the cartilage was likewise isolated by selective salt precipitation. Specimens of bone were decalcified by extraction with $0.5 \mathrm{M}$ acetic acid and collagen was solubilized by limited pepsin digestion and eventually recovered by selective salt precipitation. Neutral solvents used in the indicated extraction and purification procedures contained the protease inhibitors: ethylenediamine-tetraacetic acid (sodium salt), $50 \mathrm{mM} ; N$-ethyl-maleimide, $10 \mathrm{mM}$; phenylmethylsulfonyl fluoride, $1 \mathrm{mM}$; $\epsilon$-aminocaproic acid, 20 $\mathrm{mM}$; and benzamidine, $10 \mathrm{mM}$. Acidic solvents contained $1 \mathrm{mM}$ phenylmethylsulfonyl fluoride as well as $1 \mu \mathrm{g} / \mathrm{ml}$ pepstatin except in those instances where pepsin was used to facilitate extractions of collagen.

Collagen $\alpha$-chains were obtained from the various preparations by chromatography of the denatured collagens on agarose beads and individual $\alpha$-chains were recovered following ion-exchange chromatography on carboxymethyl (CM-)-cellulose (15) or on phosphocellulose (14). Amino acid analyses of the chains were performed following hydrolysis in $6 \mathrm{~N} \mathrm{HCl}$, as described previously (16). In certain instances, aliquots of the chain preparations were oxidized with performic acid (17) before hydrolysis and amino acid analysis. In contrast to amino acid analyses of conventional protein hydrolysates (16), analyses of oxidized chains were performed with a column temperature of $60^{\circ} \mathrm{C}$ throughout in order to allow complete resolution of $\alpha$-aminoadipic acid from glycine. Additional aliquots of selected chains were cleaved with cyanogen bromide $(\mathrm{CNBr})(18)$, and the nature and number of the cleavage products were evaluated by utilizing gel-permeation high performance liquid chromatography (HPLC), as recently described (19).

\section{Results}

Extraction and recovery of collagens. Table I presents the results obtained with respect to the extraction and recovery of the collagens from the tissues evaluated in this study. As noted in the table, dermis yielded the largest quantity of collagen, which was due to the relative abundance of the tissue. The extractability properties of the dermal collagen appeared to be well within the normal range. In this regard, the neutral salt solvent brought relatively little collagen into solution. A higher proportion of dermal collagen was solubilized in $0.5 \mathrm{M}$ acetic acid, while the majority of the collagen in this tissue was solubilized using limited pepsin proteolysis. As far as could be discerned through selective precipitation procedures as well as by characterization of the collagens (see below), the neutral salt and acetic acid extracts contained exclusively type I collagen. On the other hand, collagen solubilized by limited pepsin proteolysis represented a heterogeneous mixture of type I, III, and V collagens which were present in approximately normal proportions for a full term infant, i.e., in a ratio of $16: 3: 1$, respectively. With respect to the remaining tissues, significant quantities of collagen were solubilized only through limited proteolysis with pepsin. The collagen extracted from dura mater closely resembled that extracted from dermis, but it did not appear to contain detectable levels of type $\mathrm{V}$ collagen. The collagen solubilized by this procedure from cartilage was largely type II collagen. Nevertheless, these samples contained some type I collagen that was probably derived from contaminating bone tissue, and also contained 
Table I. Extraction and Recovery of Collagens

\begin{tabular}{|c|c|c|c|c|c|c|}
\hline \multirow[b]{2}{*}{ Tissue } & \multirow[b]{2}{*}{ Extract } & \multirow[b]{2}{*}{$\begin{array}{l}\text { Collagen } \\
\text { recovered (mg) }\end{array}$} & \multicolumn{4}{|c|}{ Proportion (\%) } \\
\hline & & & Type I & Type II & Type III & Type V \\
\hline \multirow[t]{3}{*}{ Dermis } & $1.0 \mathrm{M} \mathrm{NaCl}$ (NSSC) & 30 & 100 & - & - & - \\
\hline & $0.5 \mathrm{M} \mathrm{HAc} \mathrm{(ASC)}$ & 118 & 100 & - & - & - \\
\hline & $0.5 \mathrm{M} \mathrm{HAc}+$ pepsin (PSC) & 1,220 & 80 & - & 15 & 5 \\
\hline Dura mater & $0.5 \mathrm{M} \mathrm{HAc}+$ pepsin (PSC) & 349 & 90 & - & 10 & - \\
\hline Cartilage & $0.5 \mathrm{M} \mathrm{HAc}+$ pepsin $(\mathrm{PSC})$ & 117 & $>5$ & 90 & - & $>5$ \\
\hline Skull bones & $0.5 \mathrm{M} \mathrm{HAc}+$ pepsin $(\mathrm{PSC})$ & 120 & 98 & - & - & $>2$ \\
\hline Long bones & $0.5 \mathrm{M} \mathrm{HAc}+$ pepsin $(\mathrm{PSC})$ & 42 & 98 & - & - & $>2$ \\
\hline
\end{tabular}

some material that precipitated in a manner similar to type $\mathrm{V}$ collagen. The latter material may represent a proportion of the minor cartilage collagens that are present in cartilaginous specimens (20). The collagen present in extracts of both skull bones and long bones was predominantly type I collagen; along with it was a small proportion of type $V$ collagen. The small quantities of collagen recovered from both types of bone may be ascribed to the general lack of bone tissue in the infant. In this regard, the skull bones were present as mere patches in sporadic positions over the surface of the head, while the mass of the long bones consisted largely of granulation tissue admixed with fragments of cartilage plus cancellous bone. Therefore, relatively little uncontaminated bone could be dissected from either location.

Preparation of collagen $\alpha$-chains. The primary chain constituents of the collagens isolated from various tissues were obtained by molecular sieve chromatography on agarose beads. Fig. 2 illustrates representative chromatograms of denatured dermal type I collagen when chromatographed in this fashion. As expected, the acid soluble collagen (ASC) (Fig. 2 B) exhibited a much greater proportion of higher molecular weight components than neutral salt soluble collagen (NSSC) (Fig. $2 A$ ), which indicates that it is derived from a pool of more highly cross-linked material.

Fig. 3 presents molecular sieve chromatograms of type I collagen, which was solubilized from dermis by limited digestion with pepsin. This collagen was initially precipitated from the

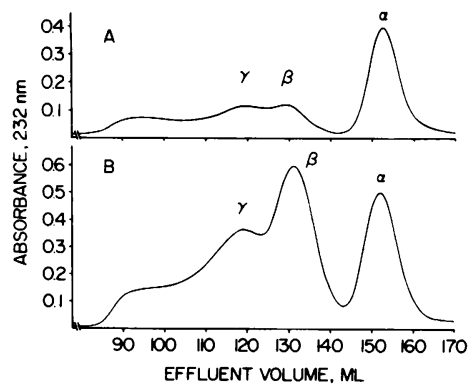

Figure 2. Agarose molecular sieve chromatography of $\operatorname{NSSC}(A)$ and ASC $(B)$ from dermis. pepsin digest at $0.7 \mathrm{M} \mathrm{NaCl}$ (13) and was followed by reprecipitation from a neutral solvent at $2.5 \mathrm{M} \mathrm{NaCl}$ (Fig. $3 \mathrm{~A}$ ) and at $3.5 \mathrm{M} \mathrm{NaCl}$ (Fig. $3 \mathrm{~B}$ ). This somewhat unusual precipitation pattern, in which a fraction of type I collagen precipitates from neutral solvent at $2.5 \mathrm{M} \mathrm{NaCl}$ and another fraction precipitates at $3.5 \mathrm{M} \mathrm{NaCl}$, has previously been observed in studies on corneal collagen (21). The present results suggest that the molecules selectively precipitated at $2.5 \mathrm{M} \mathrm{NaCl}$ (Fig. $3 A$ ) are largely in the form of aggregates cross-linked through extensive intermolecular bonding, while the more soluble molecules precipitated at $3.5 \mathrm{M} \mathrm{NaCl}$ (Fig. $3 \mathrm{~B}$ ) are largely monomeric in form and have far fewer intermolecular cross-links.

The constituent chains of other collagens isolated in the course of these studies were recovered in the same fashion, except that preparations of type III collagen were reduced and alkylated under denaturing conditions before chromatography in order to release individual $\alpha 1$ (III) chains (15). Note that in all cases, the primary chain constituents of the various collagens chromatographed in a fashion that indicated a molecular weight in the range commonly observed for collagen $\alpha$-chains, i.e., 94 95,000 mol wt.

Ion-exchange chromatography of $\alpha$-chains. In order to further characterize the collagens, their chains were resolved by chromatography on CM-cellulose. Chromatograms of the type I $\alpha$-chains that were derived from NSSC and ASC of dermis are shown in Fig. 4. The $\alpha$-chains derived from NSSC were well

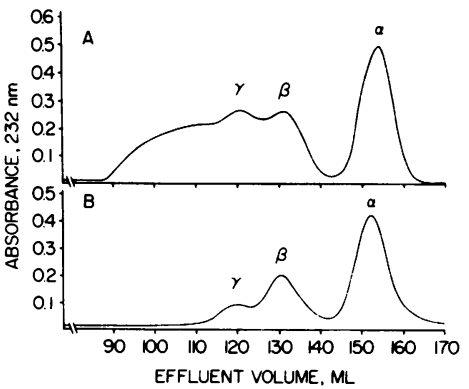

Figure 3. Agarose molecular sieve chromatography of PSC (type I) from dermis. The collagen was precipitated from the pepsin digest at $0.7 \mathrm{M} \mathrm{NaCl}$ and subsequently reprecipitated from neutral solution at $2.5 \mathrm{M}$ $\mathrm{NaCl}(A)$ and at $3.5 \mathrm{M}$ $\mathrm{NaCl}(B)$. 


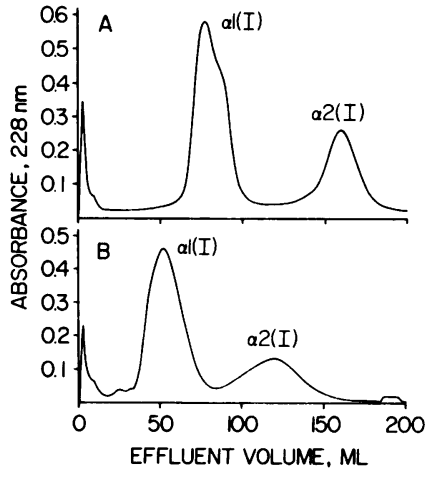

Figure 4. CM-cellulose chromatography of the type I collagen $\alpha$-chains derived from the $\operatorname{NSSC}(A)$ and ASC $(B)$ of dermis.

retained under the conditions employed and were eluted as well resolved peaks (Fig. $4 \mathrm{~A}$ ) with retention times equivalent to type I collagen chains from normal tissues that were chromatographed under the same conditions. However, $\alpha$-chains derived from ASC were less well retained by the column, and although resolution of the $\alpha 1$ (I) and $\alpha 2(\mathrm{I})$ chains was adequate (Fig. $4 \mathrm{~B}$ ) it was not as good as that observed for the chains in NSSC. Moreover, we observed similar chromatographic behavior on the part of type I chains that were derived from both fractions of pepsin-solubilized collagen (PSC) from dermis. Representative chromatograms of the chains derived from the 2.5 and $3.5 \mathrm{M}$ $\mathrm{NaCl}$ precipitates are shown in Fig. 5, $A$ and $B$, respectively. These results, which suggest a substantial deficiency in overall positive charge for the majority of the type I collagen $\alpha$-chains in dermis, were extended to type III and V collagens. The results revealed that $\alpha 1$ (III) as well as $\alpha 1(\mathrm{~V})$ and $\alpha 2(\mathrm{~V})$ chains derived from collagens that were extracted from OI dermis were likewise chromatographed on ion-exchange media in a fashion suggesting the loss of overall positive charge relative to homologous chains derived from control tissues (data not shown).

In contrast to the results described above for the chains of dermal collagens, chains from all other collagens examined in this study chromatographed similarly, if not identically, to chains derived from normal control tissues. These included the type I collagen chains of dura mater, the type II collagen chains of cartilage, as well as the type I collagen chains of both skull bones

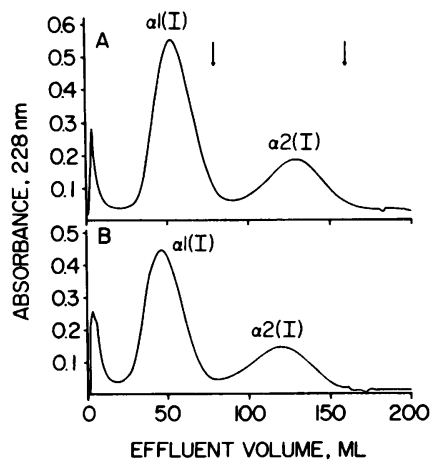

Figure 5. CM-cellulose chromatography of the type I collagen $\alpha$-chains derived from the PSC of dermis. This collagen was initially precipitated from the pepsin digest at $0.7 \mathrm{M}$ $\mathrm{NaCl}$ and was subsequently reprecipitated from neutral solution at $2.5 \mathrm{M} \mathrm{NaCl}(A)$ and at $3.5 \mathrm{M} \mathrm{NaCl}(B)$. Arrows indicate the elution positions of $\alpha 1$ (I) and $\alpha 2$ (I) chains derived from extracts of control term infant skin as well as other tissues of the $\mathrm{OI}$ infant. and long bones. A representative chromatogram obtained in the course of these studies is presented in Fig. 6; it depicts the chromatographic properties of type I collagen chains derived from PSC of dura mater. It is noteworthy in this example that the $\alpha 1(\mathrm{I})$ and $\alpha 2(\mathrm{I})$ chains of dura mater chromatograph quite similarly to the chains of neutral salt-soluble dermal collagen (Fig. $4 \mathrm{~A}$ ).

Amino acid analyses. Various collagen $\alpha$-chains, prepared as described above, were evaluated with respect to amino acid composition in an attempt to discern the reasons for the unusual chromatographic properties of the dermal chains. In general, the results indicated that the chains derived from dermal collagen were, within experimental error, identical to homologous chains from other OI tissues as well as from other normal control tissues. Nevertheless, the majority of the chains from dermal collagens showed a consistent deficiency with respect to lysyl plus hydroxylysyl residues. These data are summarized in Table II. The initial point of interest in this compilation of data is that the $\alpha 1$ (I) and $\alpha 2$ (I) chains of NSSC of the dermis contain 36 and 31 residues/1,000 of lysine (plus hydroxylysine), respectively. These values agree quite well with previous results on the type I chains of infant collagen (22). Nevertheless, the level of hydroxylysine in each chain of the NSSC is substantially higher, and the level of lysine is correspondingly lower than that observed for control dermal chains (22). In contrast, the type I chains of ASC of dermis as well as of the PSC of dermis clearly lack the full complement of lysyl and hydroxylysyl residues. The latter phenomenon is apparent when comparing the values of dermal $\alpha 1(\mathrm{III}), \alpha 1(\mathrm{~V})$, and $\alpha 2(\mathrm{~V})$ chains with homologous chains from unaffected infants $(14,15)$. It is noteworthy that the type $\mathrm{V}$ collagen chains derived from the $\mathrm{OI}$ dermis contain far fewer residues of hydroxylysine then was previously observed for these chains. The significance of this observation is not clear at present but may reflect tissue differences, since previous data on human type $\mathrm{V}$ chains has been obtained in studies on tissues other than dermis (14). The chains recovered from collagen in dura mater, cartilage, skull bones, and long bones exhibit levels of lysine (plus hydroxylysine) well within that expected for chains derived from normal tissues. These results explain the aberrant chromatographic behavior of the majority of the dermal collagen chains and indicate that their relatively acidic properties could be attributed, at least in part, to the apparent absence of five to seven lysyl (plus hydroxylysyl) residues.

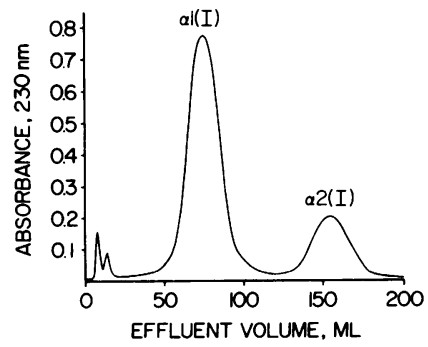

Figure 6. CM-cellulose chromatography of type I collagen $\alpha$-chains derived from PSC of dura mater. The collagen was first precipitated at $0.7 \mathrm{M}$ $\mathrm{NaCl}$ and subsequently reprecipitated from neutral solution at $2.5 \mathrm{M} \mathrm{NaCl}$. 
Table II. Partial Amino Acid Composition of Collagen Chains from OI Tissues*

\begin{tabular}{|c|c|c|c|c|c|c|c|c|c|c|c|c|c|c|c|c|}
\hline \multirow[b]{3}{*}{ Amino acid } & \multicolumn{9}{|l|}{ Dermis } & & & \multirow{3}{*}{$\begin{array}{l}\text { Cartilage } \\
\text { PSC }\end{array}$} & \multirow{2}{*}{\multicolumn{2}{|c|}{$\begin{array}{l}\text { Skull bone } \\
\text { PSC } \neq\end{array}$}} & \multirow{2}{*}{\multicolumn{2}{|c|}{$\begin{array}{l}\text { Long bone } \\
\text { PSC } \ddagger\end{array}$}} \\
\hline & \multicolumn{2}{|l|}{ NSSC } & \multicolumn{2}{|l|}{ ASC } & \multicolumn{2}{|l|}{ PSC $\ddagger$} & \multirow{2}{*}{$\frac{\text { PSC }}{\alpha 1 \text { (III) }}$} & \multicolumn{2}{|l|}{ PSC } & \multicolumn{2}{|c|}{ Dura PSC $\ddagger$} & & & & & \\
\hline & $\alpha 1(\mathrm{I})$ & $\alpha 2(\mathrm{I})$ & $\alpha 1(\mathrm{I})$ & $\alpha 2(\mathrm{I})$ & $\alpha 1(\mathrm{I})$ & $\alpha 2(\mathrm{I})$ & & $\alpha I(V)$ & $\alpha 2(\mathrm{~V})$ & $\alpha \mathrm{l}(\mathrm{I})$ & $\alpha 2(\mathrm{I})$ & & $\alpha 1(\mathrm{I})$ & $\alpha 2(\mathrm{I})$ & $\alpha 1(\mathrm{I})$ & $\alpha 2(\mathrm{I})$ \\
\hline Lysine & $28[31] \S$ & $19[23] \S$ & 23 & 17 & 21 & 15 & $25[30]^{\prime \prime}$ & $38[14] \pi$ & $20[13] \pi$ & 27 & 18 & 23 & 27 & 19 & 25 & 18 \\
\hline $\begin{array}{l}\text { Hydroxylysine } \\
\alpha \text {-Aminoadipic }\end{array}$ & $8[5]$ & $12[9]$ & 6 & 9 & 7 & 10 & $3[5]$ & $6[36]$ & $9[23]$ & 9 & 12 & 13 & 8 & 11 & 9 & 12 \\
\hline acid & - & - & (3) & (2) & (4) & $(2)$ & (2) & - & - & (0) & (0) & - & - & - & - & - \\
\hline Total & $36[36]$ & $31[32]$ & 29 & 26 & 28 & 25 & 28 [35] & $44[50]$ & $29[36]$ & 36 & 30 & 36 & 35 & 30 & 34 & 30 \\
\hline
\end{tabular}

[ ] Denote data from the previously indicated studies, i.e., data from references 14, 15, and 22. ( ) Denote values for $\alpha$-aminoadipie acid that are not included in the calculation of amino acid composition. * Values listed represent residues per 1,000 total residues rounded off to the nearest whole number. $¥$ Values listed are representative for type I collagen precipitated from neutral solvents at 2.5 and $3.5 \mathrm{M} \mathrm{NaCl}$. § Data from Reference 22 . "Data from Reference 15 . I Data from Reference 14.

In view of these results it was postulated that the lysine plus hydroxylysine "deficiency" observed in the dermal collagen samples might be due to unusually high levels of oxidative deamination, which may lead to the occurrence of high levels of allysine and hydroxyallysine. Accordingly, selected samples of the chain along with appropriate control chains were oxidized with performic acid and again examined with respect to amino acid composition. As also shown in Table II, oxidation of the apparently deficient chains with performic acid led to the recovery of two to four residues of $\alpha$-aminoadipic acid depending on the chain under investigation. In no case did the amount of $\alpha$-aminoadipic acid completely account for the apparent deficiency in lysyl plus hydroxylysyl residues. Nevertheless, $\alpha$-aminoadipic acid is the oxidation product of allysine, and the corresponding oxidation product of hydroxyallysine may have been destroyed during hydrolysis or may have chromatographed with another amino acid during the analyses. In any event, it is significant that performic acid oxidation of the control chains, i.e., type I chains derived from dura mater that exhibited no apparent deficiency in lysyl residues, did not give rise to $\alpha$-aminoadipic acid residues, as is also indicated in Table II.

$\mathrm{CNBr}$ peptide evaluations. The above results, suggesting that the chromatographic behavior of the dermal collagen OI chains could be ascribed to an unusually high level of posttranslational modification of lysyl (plus hydroxylysyl) residues, was corroborated by studies on the $\mathrm{CNBr}$ cleavage products of the chain. These results are presented in Fig. 7, which illustrates the gelpermeation HPLC chromatogram of the CNBr peptides derived from the $\alpha 1$ (I) chain of PSC from dermis. The elution pattern clearly is that which is expected for peptides derived from a typical human $\alpha 1$ (I) chain (19). Similar results were obtained for $\alpha 2$ (I) chains as well as for $\alpha 1$ (III), $\alpha 1(\mathrm{~V})$, and $\alpha 2(\mathrm{~V})$ chains derived from dermis (data not shown).

\section{Discussion}

Collagens from several tissues of one infant with lethal perinatal OI have been examined with respect to proportions of collagen types present, chain composition, compositional features of in- dividual $\alpha$-chains, and with respect to $\mathrm{CNBr}$ cleavage products of the chains. In general, the results obtained have substantiated earlier observations concerning the collagens present in selected lethal perinatal OI tissues $(7,8)$. In this regard, the proportions of collagen present in the tissue examined were apparently well within the range expected for normal tissues. Moreover, our results showed that the constituent chains of neutral-salt soluble type I collagen tended to exhibit elevated levels of hydroxylysine and, correspondingly, exhibited diminished levels of lysine when compared with collagen derived from control tissues. The most striking anomaly observed in the present study was the apparent deficiency in lysyl (plus hydroxylysyl) residues for the majority of type I collagen extracted from dermis as well as for the majority of type III and V collagens from this tissue. This apparent deficiency, which also manifested itself in the chromatographic behavior of the chains, could be correlated with the presence of unusually high levels of allysyl residues in the indicated chains. The latter residues were detected as residues of $\alpha$-aminoadipic acid after performic acid oxidation and hydrolysis of the chains. Although the formation of allysyl and hydroxyallysyl residues is required to initiate cross-linking reactions in collagen fibers, these residues are largely confined to the nonhelical extremities of individual collagen chains and are therefore generally present at the level of two residues per chain (23). Our finding, that

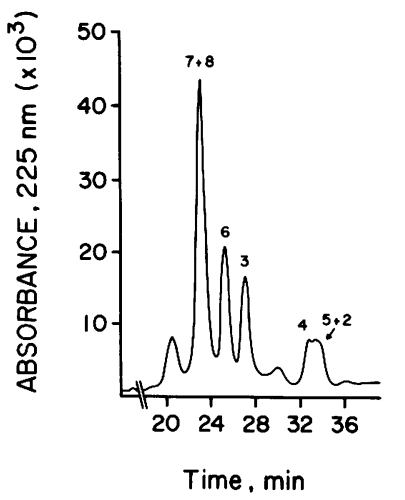

Figure 7. Gel-permeation HPLC of the $\mathrm{CNBr}$ peptides derived from the $\alpha 1$ (I)-chain recovered from PSC of the OI dermis. 
chains of PSC of the OI dermis contain some two to four residues of allysine, indicates that a significant number of residues within the potentially helical portion of the chains are oxidatively deaminated. As noted above, this is likely to be a conservative estimate of the number of oxidative deamination reactions because of uncertainties regarding the detection of the performic acid oxidation product of hydroxyallysine. And finally, evaluation of the $\mathrm{CNBr}$ peptide patterns of the chains derived from the OI infant's dermal collagen revealed that the alterations observed were not likely to be due to gross structural aberrations. The results outlined above are compatible with the notion that the basic defect in collagen metabolism in the OI infant investigated in this study is an unusually high level of posttranslational modifications of structurally normal chains. The defects would thus appear to be transmitted through a recessive mode of inheritance. This mode of inheritance has been proposed to be the mode in which lethal perinatal OI is transmitted (1).

The results presented here, along with the results of previous studies $(7,8)$, raise several questions concerning the relationship between the biochemical anomalies and the clinical manifestations of the disorder. It has been proposed that the formation of collagen fibers from molecules containing elevated hydroxylysine levels might, in itself, be inhibitory of subsequent mineralization and of the elaboration of a calcified tissue (7, 8). Alternatively, it has been suggested that increased glycosylation accompanying elevated hydroxylysine levels may interfere with normal fiber formation and with subsequent mineral deposition $(7,8)$. Our results favor the latter possibility. In this regard, the most efficient substrates for lysyl oxidase are preparations of native collagen fibers (24). Therefore, the evidence for increased lysyl oxidase activity observed in the present studies could ultimately be due to an aberrant fibril form that was conducive to extensive lysyl oxidase activity. These modifications could, in turn, exacerbate defective fiber formation, since they would be expected to disrupt the normal charge-charge interactions accompanying the juxtaposition of microfibrils and the formation of major fibrous elements.

It is, perhaps, more difficult to explain why the deficiency in lysine plus hydroxylysine residues was not observed in collagen extracted from cartilage, dura mater, and even from bone, which was so severely affected clinically. It may be considered that the physiological consequences of the presence of aberrant fiber forms in dermis are not so great as in bone, where mechanical stresses are greater and where the requirements for mineral deposition impose an even greater need for precision in alignment of molecules within fibers. Thus, the abnormal collagen fibers might be eliminated more rapidly from bone than from dermis. There is, at present, no evidence for such a mechanism, yet it would adequately account for the severely low mass of bone encountered in the infant. In accordance with the foregoing discussion, the apparent lack of elevated levels of allysine or hydroxyallysine in collagens from cartilage and dura mater could be ascribed to the presence of isozymes with respect to lysyl hydroxylase, the hydroxylysine glycosylating enzymes, as well as to lysyl oxidase. Although data on lysyl hydroxylylase (25) and collagen glucosyltransferase (26) in various chick embryo tissues indicate that these enzymes exhibit biochemical and immunochemical identity regardless of tissue source, there is a precedent for the presence of lysyl hydroxylase isozymes in human tissues, since hydroxylysine deficiency in the type VI Ehlers-Danlos syndrome is tissue specific $(27,28)$.

The present communication represents the first report of an alteration in collagen metabolism in an inherited disorder leading to the elaboration of fibrous elements containing unusually high levels of aldehyde functions derived through oxidative deamination of lysyl, and, presumably, hydroxylysyl residues. Nevertheless, a similar phenomenon may occur during the administration of D-penicillamine to experimental animals (29). In retrospect, our observations may aid in explaining certain previous results concerning collagen and its metabolism in OI. Thus, formation of a doublet peak for $\alpha 1(\mathrm{I})$ and the apparent absence of an $\alpha 2$ (I) chain in the collagen synthesized by fibroblasts from an OI-like patient (30) could very well be explained by alteration of the ion-exchange properties of at least a portion of the respective chains, as observed in the present studies. In addition, the unusual aggregation properties of type I collagen isolated from the cells of an OI patient (31) could be ascribed to an unusual number of highly reactive aldehyde functions in the molecules. In any event, the finding of above-normal levels of lysyl-derived aldehyde functions in the collagen of this one infant with lethal perinatal OI amplifies the growing list of reported defects in this form of OI $(7,8,11,12)$, and further emphasizes the biochemical as well as the clinical heterogeneity of this syndrome.

\section{Acknowledgments}

The authors would like to express their gratitude to Ms. Melody Edwards, who assisted in all phases of the preparation of the manuscript, Ms. Margaret Vail, for technical assistance, and Dr. John J. Sauk of the University of Minnesota, Minneapolis, MN, who arranged for acquisition and delivery of the tissues. This work was supported by DHHS grant DE-02670 as well as by a grant from the Osteogenesis Imperfecta Foundation, Inc.

\section{References}

1. Sillence, D. O., A. S. Senn, and D. M. Danks. 1979. Genetic heterogeneity in osteogenesis imperfecta. J. Med. Genet. 16:101-116.

2. Hollister, D. W. 1981. Osteogenesis imperfecta: promising beginnings and continuing challenges. Collagen Relat. Res. 1:228-234.

3. Miller, E. J., and O. M. Petrovic. 1981. The organic matrix in congenital bone disease-a look toward the future. In The Chemistry and Biology of Mineralized Connective Tissues. A. Veis, editor. Elsevier/ North-Holland, New York. 207-212.

4. Byers, P. H., G. S. Barsh, K. E. Peterson, K. A. Holbrook, and D. W. Rowe. 1981. Molecular mechanisms of abnormal bone matrix formation in osteogenesis imperfecta. In The Chemistry and Biology of Mineralized Connective Tissues. A. Veis, editor. Elsevier/North-Holland, New York. 213-222.

5. Byers, P. H., G. S. Barsh, and K. A. Holbrook. 1982. Molecular pathology in inherited disorders of collagen metabolism. Hum. Pathol. 13:89-95. 
6. Eastoe, J. E., P. Martins, and N. R. Thomas. 1973. The aminoacid composition of human hard tissue collagens in osteogenesis imperfecta and dentinogenesis imperfecta. Calcif. Tissue Res. 12:91-100.

7. Trelstad, R. L., D. Rubin, and J. Gross. 1977. Osteogenesis imperfecta congenita. Evidence for a generalized molecular disorder of collagen. Lab. Invest. 36:501-508.

8. Kirsch, E., T. Krieg, K. Remberger, H. Fendel, P. Bruckner, and P. K. Müller. 1981. Disorder of collagen metabolism in a patient with osteogenesis imperfecta (lethal type): increased degree of hydroxylation of lysine in collagen types I and III. Eur. J. Clin. Invest. 11:39-47.

9. Pope, F. M., A. C. Nicholls, C. Eggleton, P. Nircissi, E. Hey, and J. M. Parkin. 1980. Osteogenesis imperfecta (lethal) bones contain types III and V collagens. J. Clin. Pathol. (Lond.). 33:534-538.

10. Penttinen, R. P., J. R. Lichtenstein, G. R. Martin, and V. A. McKusick. 1975. Abnormal collagen metabolism in cultured cells in osteogenesis imperfecta. Proc. Natl. Acad. Sci. USA. 72:586-589.

11. Barsh, G. S., and P. H. Byers. 1981. Reduced secretion of structurally abnormal type I procollagen in a form of osteogenesis imperfecta. Proc. Natl. Acad. Sci. USA. 78:5142-5146.

12. Williams, C. J., and D. J. Prockop. 1983. Synthesis and processing of a type I procollagen containing shortened pro- $\alpha$ l(I) chains by fibroblasts from a patient with osteogenesis imperfecta. J. Biol. Chem. 258:59155921.

13. Miller, E. J., and R. K. Rhodes. 1982. Preparation and characterization of the different types of collagen. Methods Enzymol. 82A:3364.

14. Rhodes, R. K., and E. J. Miller. 1978. Physicochemical characterization and molecular organization of the collagen A and B chains. Biochemistry. 17:3442-3448.

15. Chung, E., and E. J. Miller. 1974. Collagen polymorphism: characterization of molecules with the chain composition $[\alpha 1(\mathrm{III})]_{3}$ in human tissues. Science (Wash. DC). 183:1200-1201.

16. Miller, E. J. 1972. Structural studies on cartilage collagen employing limited cleavage and solubilization with pepsin. Biochemistry. 11:4903-4909.

17. Moore, S. 1962. On the determination of cystine as cysteic acid. J. Biol. Chem. 238:235-237.

18. Miller, E. J., E. H. Epstein, Jr., and K. A. Piez. 1971. Identification of three genetically distinct collagens by cyanogen bromide cleavage of insoluble human skin and cartilage collagen. Biochem. Biophys. Res. Commun. 42:1024-1029.

19. Miller, E. J., R. K. Rhodes, and D. K. Furuto. 1983. Identification of collagen chains as a function of cyanogen bromide peptide patterns using gel permeation high-performance liquid chromatography. Collagen Relat. Res. 3:79-87.

20. Burgeson, R. E., P. A. Hebda, N. P. Morris, and D. W. Hollister. 1982. Human cartilage collagens. Comparison of cartilage collagens with human type V collagen. J. Biol. Chem. 257:7852-7856.

21. Welsh, C., S. Gay, R. K. Rhodes, R. Pfister, and E. J. Miller. 1980. Collagen heterogeneity in normal rabbit cornea. I. Isolation and biochemical characterization of the genetically-distinct collagens. Biochim. Biophys. Acta. 625:78-88.

22. Epstein, E. H., Jr., R. D. Scott, E. J. Miller, and K. A. Piez. 1971. Isolation and characterization of the peptides derived from soluble human and baboon skin collagen after cyanogen bromide cleavage. $J$. Biol. Chem. 246:1718-1724.

23. Miller, E. J. 1984. Chemistry of the collagens and their distribution. In Connective Tissue Biochemistry. K. A. Piez and H. Reddi, editors. Elsevier/North-Holland, New York. In press.

24. Siegel, R. C. 1974. Biosynthesis of collagen crosslinks: increased activity of purified lysyl oxidase with reconstituted collagen fibers. Proc. Natl. Acad. Sci. USA. 71:4826-4830.

25. Turpeenniemi-Hujanen, T. M. 1981. Immunological characterization of lysyl hydroxylase, an enzyme of collagen synthesis. Biochem. J. 195:669-676.

26. Myllylä, R. 1981. Preparation of antibodies to chick-embryo galactosylhydroxylysyl glucosyltransferase and their use for an immunological characterization of the enzyme of collagen synthesis. Biochim. Biophys. Acta. 658:299-307.

27. Pinnell, S. R., S. M. Krane, J. E. Kenzora, and M. J. Glincher. 1972. A heritable disorder of connective tissue: hydroxylysine deficient collagen disease. N. Engl. J. Med. 286:1013-1020.

28. Hanauske-Abel, H. H., and K. H. Rohm. 1980. The collagenous part of $\mathrm{Clq}$ is unaffected in the hydroxylysine-deficient collagen disease. FEBS (Fed. Eur. Biochem. Soc.) Lett. 110:73-76.

29. Deshmukh, K., and M. E. Nimni. 1969. A defect in the intramolecular and intermolecular cross-linking of collagen caused by penicillamine. II. Functional groups involved in the interaction process. $J$. Biol. Chem. 244:1787-1795.

30. Meigel, W. N., P. K. Müller, B. F. Pontz, N. Sorensen, and J. Spranger. 1974. A constitutional disorder of connective tissue suggesting a defect in collagen biosynthesis. Klin. Wochenschr. 52:906-912.

31. Peltonen, L., A. Palotie, T. Hayashi, and D. J. Prockop. 1980. Thermal stability of type I and type III procollagens from normal human fibroblasts and from a patient with osteogenesis imperfecta. Proc. Natl. Acad. Sci. USA. 77:162-166. 